\title{
Hospital-based surveillance of meningococcal meningitis in Salvador, Brazil
}

\author{
Soraia M. Cordeiro ${ }^{a}$, Alan B. Neves ${ }^{a}$, Cássio T. Ribeiro ${ }^{a}$, Maya L. Petersen $^{b}$, \\ Edilane L. Gouveia ${ }^{a}$, Guilherme S. Ribeiro ${ }^{a}$, Tatiana S. Lôbo ${ }^{a}$, \\ Joice N. Reis ${ }^{a, c}$, Kátia M. Salgado ${ }^{d}$, Mitermayer G. Reis ${ }^{a}$, Albert I. Ko ${ }^{a, e, *}$
}

\author{
a Gonçalo Moniz Research Center, Oswaldo Cruz Foundation, Brazilian Ministry of Health, Rua Waldemar Falcão 121, Salvador, \\ Bahia 40296-710, Brazil \\ b Joint Medical Program/Division of Epidemiology, School of Public Health, University of California, Berkeley, CA, USA \\ c School of Pharmacy, Federal University of Bahia, Salvador, Brazil \\ ${ }^{d}$ Couto Maia Hospital, Bahia Secretary of Health, Salvador, Brazil \\ e Division of International Medicine and Infectious Diseases, Weill Medical College of Cornell University, New York, USA
}

Received 21 March 2007; received in revised form 14 June 2007; accepted 14 June 2007

Available online 6 August 2007

\section{KEYWORDS \\ Meningococcal \\ disease; \\ Meningitis; \\ Neisseria \\ meningitidis; \\ Serogroup; \\ Epidemiology; \\ Brazil}

\begin{abstract}
Summary This study aimed to describe the clinical, epidemiological and microbiological features of meningococcal meningitis in Salvador, Brazil. Between February 1996 and January 2001, a hospital-based surveillance prospectively identified cases of culture-positive meningococcal meningitis. Demographic and clinical data were collected through interview and medical chart review. Antisera and monoclonal antibodies were used to determine the serogroup and serotype:serosubtype of the isolates, respectively. Surveillance identified a total of 408 cases of meningococcal meningitis, with a case fatality rate of $8 \%(32 / 397)$. The mean annual incidence for the 304 culture-positive cases residing in metropolitan Salvador was 1.71 cases per 100000 population. Infants $<1$ year old presented the highest incidence (14.7 cases per 100000 population). Of the 377 serogrouped isolates, $82 \%, 16 \%, 2 \%$ and $0.3 \%$ were serogroups B, C, W135 and $\mathrm{Y}$, respectively. A single serotype:serosubtype $(4,7: \mathrm{P} 1.19,15)$ accounted for $64 \%$ of all cases. Continued surveillance is necessary to characterise strains and to define future prevention and control strategies.

(c) 2007 Royal Society of Tropical Medicine and Hygiene. Published by Elsevier Ltd. All rights reserved.
\end{abstract}

\section{Introduction}

Neisseria meningitidis is a common cause of disease worldwide, responsible for significant morbidity and mortality in infants and young children (Bilukha and Rosenstein, 2005). Without appropriate antimicrobial treatment, most cases
* Corresponding author. Tel.: +55 713176 2302; fax: +55 7131762281 .

E-mail address: aik2001@med.cornell.edu (A.I. Ko). 
of meningococcal meningitis are fatal (Flexner, 1913) and even with prompt intervention the case fatality rate (CFR) reaches 10\% (Caugant, 1998). Among those who survive, permanent sequelae, including deafness, cognitive impairment and paralysis, are common (Edwards and Baker, 1981; Kirsch et al., 1996).

In 1999, the UK introduced a highly efficacious proteinconjugate vaccine against $N$. meningitidis serogroup C (Trotter et al., 2004). Six years later, a tetravalent conjugate meningococcal vaccine, including the capsular polysaccharides of serogroups $A, C, Y$ and $W 135$, was licensed for use among persons aged $11-55$ years (Bilukha and Rosenstein, 2005). Despite these advances, an effective vaccine against serogroup B, a major cause of invasive meningococcal disease in many European countries and the Americas, is not currently available (Bjune et al., 1991; De Moraes et al., 1992; Sierra et al., 1991; Tappero et al., 1999; Tsolia et al., 2006). Future prevention of serogroup B disease will rely both on outer membrane vesicle (OMV) vaccines being used for specific serosubtypes and new vaccines containing multiple other antigens.

In Brazil, serogroup B meningococcal disease has been endemic since the 1980s and is associated with the majority of meningococcal disease. Furthermore, specific serotype:serosubtype combinations of the ET-5 complex, including $\mathrm{B}: 4,7: \mathrm{P} 1.19,15$, have been identified as the predominant serogroup $B$ strain both in epidemic and endemic situations (Sacchi et al., 1992, 2001).

Data on the epidemiology of meningococcal meningitis from population-based surveillance are necessary to characterise better circulating strains and to guide decisions regarding future prevention and control strategies for meningococcal meningitis. However, prior studies in Brazil were based on notification reports and on isolate collections. In the present study, we report the clinical, epidemiological and microbiological findings of a 5-year active hospitalbased surveillance for meningococcal meningitis in Salvador, Brazil.

\section{Materials and methods}

\subsection{Surveillance}

From 1 February 1996 to 31 January 2001, active hospital-based surveillance for meningococcal meningitis was performed in Couto Maia Hospital, the state reference hospital for infectious diseases in Salvador, Brazil. Currently, metropolitan Salvador is the third largest urban area in Brazil, with a population of 2.6 million inhabitants (Brazilian Institute for Geography and Statistics, 2002). As part of the State Health Department protocol, all suspected cases of meningitis should be referred to this hospital, where diagnostic evaluation, including lumbar puncture and cerebrospinal fluid (CSF) analysis, is performed at the emergency room. Notification of meningitis cases to state health officials is mandatory and the study hospital reports $98 \%$ of the cases among residents of metropolitan Salvador. After the initial evaluation, patients with evidence of meningitis are admitted. Rarely, they are transferred to another facility.

During the surveillance period, project personnel reviewed the clinical laboratory results 5 days a week to identify culture-confirmed cases of meningococcal meningitis. All patients with clinical signs and symptoms of meningitis and CSF culture positive for $N$. meningitidis were entered into the study according to the study protocol.

A standardised data entry form was administered for each case, which collected information on demographics, clinical history and laboratory findings during patient interviews and medical chart review. Physicians routinely performed neurological examinations and assessment of sequelae during hospitalisation. Information on sequelae present at the time of hospital discharge was analysed.

\subsection{Laboratory methods}

Neisseria meningitidis isolates from CSF cultures were incubated overnight at $37^{\circ} \mathrm{C}$ on chocolate agar in a $\mathrm{CO}_{2}$ atmosphere. Coagglutination with specific antisera was used to identify the isolate serogroup (Difco Laboratories, Detroit, MI, USA). Monoclonal antibodies were used to identify isolate serotype and serosubtype, using the dot-blot method as described previously (Wedege et al., 1990).

\subsection{Statistical analysis}

Patients residing in the city of Salvador or in one of the 29 municipalities located within $75 \mathrm{~km}$ of Salvador (aggregate population of 3.5 million) were included for incidence calculations. The surveillance hospital is the only infectious disease reference centre within this metropolitan area. Incidence calculations were based on the 2000 Brazilian census, provided by the Brazilian Institute for Geography and Statistics without adjusting for population growth (Brazilian Institute for Geography and Statistics, 2002).

Data were entered and managed by Epi Info version 6.04 (CDC, Atlanta, GA, USA) and analysed with SAS for Windows version 8.02 (SAS Institute, Cary, NC, USA) and Epi Info version 6.04 . Ratios and $95 \% \mathrm{Cl}$ were calculated by the Mid-P algorithm (Berry and Armitage, 1995). Statistical significance for comparison of proportions was assessed by $\chi^{2}$ test or, when the expected cell value was $<5$, by Fisher's exact test. Median values were compared by the Kruskal-Wallis method. Differences were considered statistically significant when the two-tailed $P$-value was $<0.05$.

\section{Results}

During the 5-year study period of active hospital-based surveillance, 408 cases of meningococcal meningitis were identified. The monthly number of cases of meningococcal meningitis varied by season, with the highest proportion of cases observed during the months of increased rainfall in the fall and winter and the lowest proportion of cases occurring during the summer (Figure 1).

Epidemiological data were available for 397 (97\%) of the cases. The CFR was $8 \%(32 / 397)$ and neurological sequelae occurred in $3 \%(11 / 365)$ of survivors at the time of hospital discharge (Table 1). The CFR was significantly higher in cases presenting with petechiae $(11 \%$ vs. $3 \% ; P=0.003)$ and in cases with a hospital course including seizures $(15 \%$ vs. $6 \% ; P=0.04)$. Blood cultures were performed for $28 \%$ of the patients $(112 / 397)$, of whom $31 \%(35 / 112)$ had positive 


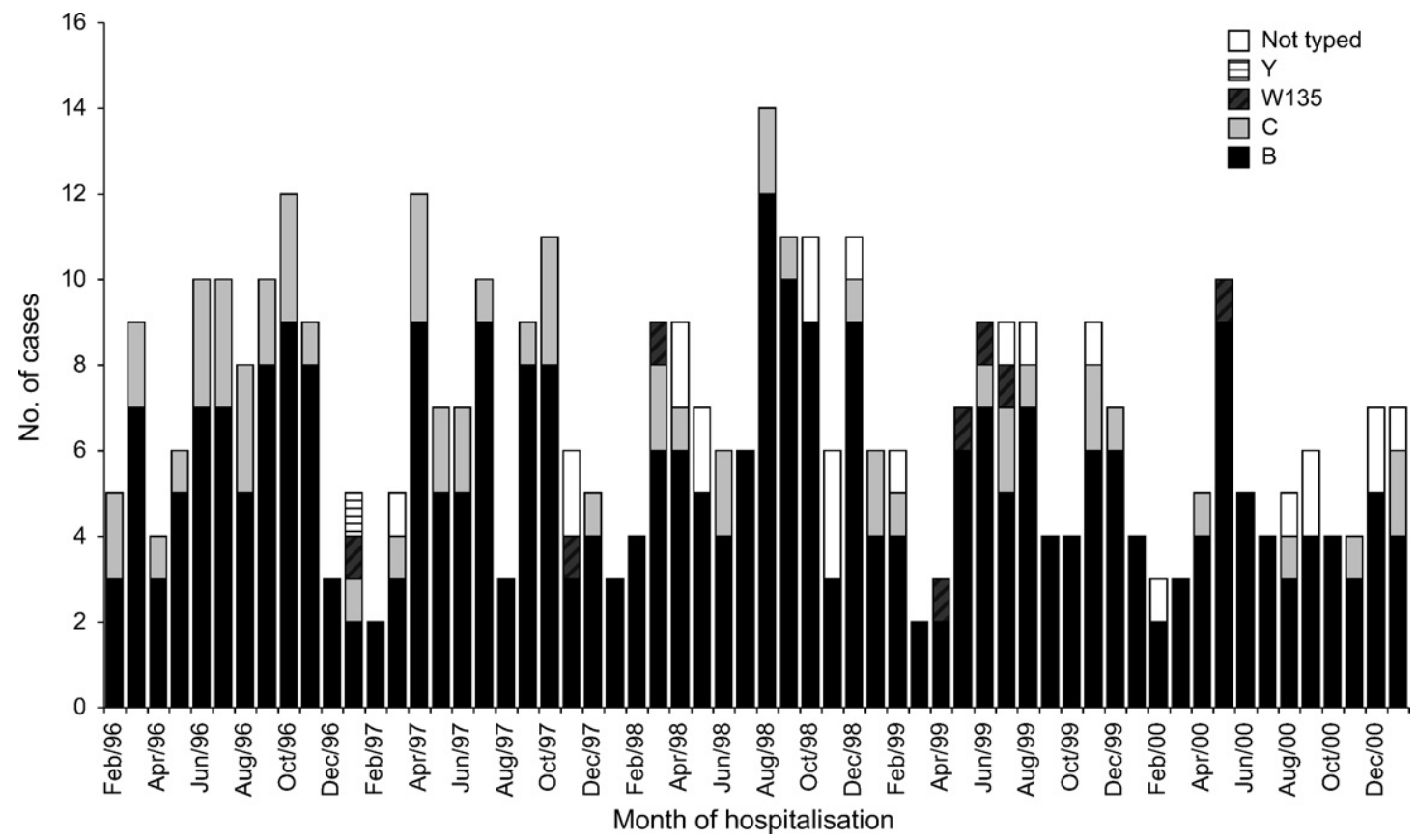

Figure 1 Distribution of meningococcal meningitis cases according to serogroup and month of identification during active hospitalbased surveillance in Salvador, Brazil.

cultures for $N$. meningitidis. Patients with positive blood culture results had a higher CFR $(17 \%$ vs. $5 \%$; $P=0.06)$.

The mean annual incidence of meningococcal meningitis, based on the 304 culture-positive cases who were residents of metropolitan Salvador, was 1.71 cases per 100000 population between February 1996 and January 2001 (Table 2). During the same period, the incidence of culture-positive Streptococcus pneumoniae and Haemophilus influenzae meningitis was 1.38 and 2.05 cases per 100000 population, respectively. Overall annual incidences of meningococcal meningitis were $2.00,1.55,2.23,1.55$ and 1.24 per 100000 population in Years 1 through 5 , respectively ( $\chi^{2}$ test for trend, $P=0.029)$. The age-specific incidence was highest in children $<1$ year of age $(14.7 / 100000)$, peaking at age 4-5 months (29.4/100000) and declining with increasing age thereafter (Table 2).

Laboratory data were available for 377 (92\%) of the cases. Serogroup B was the most frequent serogroup (309/377; 82\%), followed by serogroup C $(60 / 377 ; 16 \%)$, W135 (7/377; $2 \%)$ and $Y(1 / 377 ; 0.3 \%)$ (Table 1$)$. The most frequent serotype:serosubtype was 4,7:P1.19,15, followed by $4,7: \mathrm{P} 1.7,1$, then $4,7: \mathrm{P} 1.3$ and $2 \mathrm{a}: \mathrm{P} 1.2$, which were identified in $63.7 \%, 3.7 \%, 2.7 \%$ and $2.4 \%$ of cases, respectively (Table 3).

The median age of patients with serogroups B, C and W135 disease was 7, 10 and 9 years, respectively. Patients with serogroup $C$ disease were more likely to be $>2$ years of age compared with other serogroup infections $(87 \%$ vs. $73 \% ; P=0.03$ ), whilst patients with serogroup $B$ isolates were more likely to be $<2$ years old $(27 \%$ vs. $15 \% ; P=0.05)$.

\section{Discussion}

This study describes the clinical, epidemiological and microbiological features of meningococcal meningitis in Salvador,
Brazil. Active hospital-based surveillance was performed in metropolitan Salvador and detected a mean annual incidence of 1.71 cases of meningococcal meningitis per 100000 population, with a CFR of $8.1 \%$. These rates are comparable with those seen in the USA and Europe (Bilukha and Rosenstein, 2005; Rosenstein et al., 1999, 2001). As a caveat, rates of culture-positive meningococcal meningitis are likely to underestimate the true disease burden, which is expected to be higher than that encountered in developed countries, since case ascertainment may not have been complete and use of antibiotics prior to hospitalisation may have interfered with the efficiency of culture isolation.

Our data support previous observations where the incidence of meningococcal meningitis was higher in young children, peaking in infants $4-5$ months of age (Peltola, 1983; Rosenstein et al., 1999). However, in the USA and Europe, an elevated rate of meningococcal disease has been reported among teenagers and young adults (Cartwright et al., 2001; Rosenstein et al., 1999). In this situation, increased risk was associated with exposure to diverse strains of $N$. meningitidis during college attendance, especially for students residing in dormitories (Cartwright et al., 2001; Rosenstein et al., 2001). In contrast, meningococcal meningitis cases in Salvador were predominantly members of poor slum (favela) communities. A large proportion of the population is presumably exposed early in life owing to high endemic transmission of meningococcal strains in these densely populated communities. The lower attack rates observed among adolescents and adults in comparison with infants may relate to early acquisition of naturally acquired immunity in this high transmission setting.

Increased numbers of meningococcal cases tended to occur during the fall and winter months in Salvador, which is situated in the Southern Hemisphere tropics and has a mean daily relative humidity of $75-90 \%$. The fall and 
Table 1 Characteristics of the Neisseria meningitidis cases identified during active hospital-based surveillance in Salvador, Brazil, between February 1996 and January 2001

\begin{tabular}{|c|c|c|}
\hline Characteristic & Total no. of responses & No. (\%) of patients ${ }^{a}$ \\
\hline \multicolumn{3}{|l|}{ Demographic data } \\
\hline Male & 397 & $223(56)$ \\
\hline Age $0-2$ years & 397 & $98(25)$ \\
\hline Median (range) age (years) & & $8(0-66)$ \\
\hline \multicolumn{3}{|l|}{ Clinical presentation } \\
\hline Fever & 325 & $319(98)$ \\
\hline Nausea or vomiting & 325 & $268(82)$ \\
\hline Purpura or petechiae & 342 & $141(41)$ \\
\hline Nuchal rigidity & 326 & $134(41)$ \\
\hline Seizures & 326 & $51(16)$ \\
\hline Neurological focal signs & 281 & $13(5)$ \\
\hline \multicolumn{3}{|l|}{ Hospital course } \\
\hline Seizures & 373 & $41(11)$ \\
\hline ICU admission & 377 & $53(14)$ \\
\hline \multicolumn{3}{|l|}{ Laboratory data } \\
\hline \multicolumn{3}{|l|}{ CSF examination } \\
\hline Median (range) leukocyte count $\left(\times 10^{3} / \mu \mathrm{l}\right)$ & 393 & $10.0(7.0-21.2)$ \\
\hline Median (range) glucose level (mg/dl) & 394 & $20(0-220)$ \\
\hline Median (range) protein level (mg/dl) & 392 & $300(0-800)$ \\
\hline Positive blood culture & 112 & $35(31)$ \\
\hline \multicolumn{3}{|l|}{ Serogroup } \\
\hline B & 377 & $309(82)$ \\
\hline C & 377 & $60(16)$ \\
\hline W135 & 377 & $7(2)$ \\
\hline $\mathrm{Y}$ & 377 & $1(0.3)$ \\
\hline \multicolumn{3}{|l|}{ Clinical outcome } \\
\hline Median (range) days of hospitalisation & 383 & $11(0-43)$ \\
\hline Neurological sequelae on hospital discharge ${ }^{b}$ & 323 & $11(3)$ \\
\hline No. of deaths (case fatality rate (\%)) & 397 & $32(8)$ \\
\hline
\end{tabular}

ICU: Intensive Care Unit; CSF: cerebrospinal fluid.

a Data are No. (\%) of patients, unless otherwise indicated.

b Sequelae among 323 survivors with available information included ataxia (5), auditory deficit (3), motor deficit (2) and cognitive deficit (1).

winter seasons occur between April and September and correspond to the seasonal period of increased rainfall and cooler temperatures. However, variation in temperature is small throughout the year with mean temperatures ranging between $25^{\circ} \mathrm{C}$ and $34^{\circ} \mathrm{C}$. In temperate zones in the Northern Hemisphere, seasonal increases in meningococcal cases occur during the winter and spring (Jackson and Wenger, 1993). Seasonality may vary from place to place depending on other risk factors such as age and meningococcal phenotype (Jensen et al., 2003). The observed seasonal increase in cases in Salvador may relate to rainfall and, in turn, to increased household crowding during these periods, which facilitates transmission. However, evaluation of seasonal patterns and the influence of climactic factors will require the use of appropriate analytical approaches such as time-series modelling.

Serogroup B meningococcus emerged in the 1990s to become the major cause of meningococcal meningitis in Salvador. Eighty-two percent of cases were caused by serogroup $B$ strains and $64 \%$ of all cases were caused by a single serosubtype, $\mathrm{P} 1.19,15$. These findings confirm results from passive surveillance performed throughout Brazil in 2001, in which $67 \%$ of cases were caused by serogroup B and $54 \%$ were caused by serosubtype B:4,7:P1.19,15 (Lemos et al., 2006). The high predominance of a single serogroup and serosubtype and the moderately high incidence sustained throughout the study period are characteristic of extended outbreaks, as described in Latin America and other parts of the world following introduction and establishment of strains from the ET-5 complex (Caugant, 1998; Caugant et al., 1987; Diermayer et al., 1999; Poolman et al., 1986; Sacchi et al., 2001).

The overwhelming dominance of serogroup B disease shows that there is no indication for administration of a capsular vaccine against serogroups $A$ and $C$ in Salvador at this time. Continued follow-up in the same hospital revealed that to date serogroup $B$ has remained the prominent serogroup in Salvador, Brazil (data not shown). However, the incidence of serogroup $C$ has increased in most regions of Brazil since 2002, mainly in the southeastern area 
Table 2 Annual incidence of meningococcal meningitis and case fatality rates (CFR) by age group and year during active surveillance in Salvador, Brazil, between February 1996 and January $2001^{\text {a }}$

\begin{tabular}{|c|c|c|c|c|c|c|c|c|}
\hline \multirow{2}{*}{$\begin{array}{l}\text { Age group } \\
\text { (years) }\end{array}$} & \multirow[t]{2}{*}{ No. of cases } & \multirow{2}{*}{$\begin{array}{l}\text { No. (\%) of } \\
\text { deaths (CFR) }\end{array}$} & \multicolumn{5}{|c|}{ Annual incidence ${ }^{b}$} & \multirow[t]{2}{*}{ Average incidence } \\
\hline & & & 1996 & 1997 & 1998 & 1999 & 2000 & \\
\hline$<1$ & 47 & $5(10.6)$ & 20.3 & 15.6 & 9.4 & 14.1 & 14.1 & 14.7 \\
\hline $1-2$ & 35 & $2(5.7)$ & 3.9 & 5.4 & 10.1 & 2.3 & 5.4 & 5.4 \\
\hline $3-4$ & 30 & $1(3.3)$ & 8.7 & 0.8 & 6.3 & 5.5 & 2.4 & 4.7 \\
\hline $5-9$ & 55 & $5(9.1)$ & 2.8 & 3.5 & 5.0 & 2.5 & 3.5 & 3.5 \\
\hline $10-14$ & 52 & $4(7.7)$ & 3.5 & 4.1 & 3.5 & 2.0 & 2.0 & 3.0 \\
\hline $15-19$ & 40 & $2(5.0)$ & 2.4 & 1.4 & 2.9 & 1.9 & 1.0 & 1.9 \\
\hline $20-29$ & 23 & $3(13.0)$ & 0.6 & 0.6 & 1.3 & 0.6 & 0.3 & 0.7 \\
\hline $30-39$ & 12 & $1(8.3)$ & 0.7 & 0.2 & 0.4 & 0.7 & 0.2 & 0.4 \\
\hline $40-49$ & 4 & $2(50.0)$ & 0.5 & 0.2 & 0.0 & 0.2 & 0.0 & 0.2 \\
\hline $50-59$ & 1 & $0(0)$ & 0.4 & 0.0 & 0.0 & 0.0 & 0.0 & 0.1 \\
\hline$\geq 60$ & 1 & $0(0)$ & 0.0 & 0.0 & 0.4 & 0.0 & 0.0 & 0.1 \\
\hline All ages & 300 & $25(8.3)$ & 2.00 & 1.55 & 2.23 & 1.55 & 1.24 & 1.71 \\
\hline
\end{tabular}

a The table encompasses cases from the metropolitan region of Salvador $(n=304)$ less 4 cases whose ultimate outcome was unknown.

b Cases per 100000 inhabitants. In 1996, all incidence data were corrected to represent a 12-month period.

(SVS/MS, 2005). Thus, continued surveillance is necessary to detect possible changes in the meningococcal meningitis pattern.

Owing to the poor immunogenicity of the serogroup $B$ capsular polysaccharide, attempts to develop a vaccine protecting against serogroup $B$ disease have involved many bacterial components, including the proteins determining type and subtype specificity. An effective OMV-based vaccine against the dominant serosubtypes present in Brazil could prevent significant morbidity and mortality. The OMV vaccines can be 'tailor-made' and current approaches to vaccine development have been based on preparations derived from strains such as $B: 4,7: P 1.19,15$, which are the most predominant invasive strains in high endemicity settings. To address the potential usefulness of such vaccines, it is important to monitor the circulating serogroup $B$ phenotypes as defined by serotype:serosubtype patterns (Lind and Berthelsen, 2005).

A hexavalent OMV-based vaccine developed in The Netherlands against six common serosubtypes (P1.19,15, $\mathrm{P} 1.7,1, \mathrm{P} 1.7,4, \mathrm{P} 1.5,2, \mathrm{P} 1.5,10$ and $\mathrm{P} 1.12,13)$ appears to be safe and immunogenic in adults and older children (Pollard and Levin, 2000). This vaccine, which includes strains with serosubtypes that account for $70.6 \%$ of cases of meningococcal meningitis in Salvador, would be a candidate for use in Salvador if it proves efficacious. However, existing limitations of OMV-based meningococcal vaccines, including lower efficacy in young children (Costa et al., 1996; De Moraes et al., 1992; Sierra et al., 1991; Tappero et al., 1999), inability to induce immunological memory (Wedege et al., 1998) and failure to provide cross-protection against non-vaccine strains, may delay their use (Pollard and Levin, 2000). Alternatively, reverse vaccinology approaches have identified antigens that induce antibactericidal antibody responses against the spectrum of meningococcal serogroup B strains associated with invasive disease (Giuliani et al., 2006; Masignani et al., 2003). These target antigens are being evaluated as candidates for a universal serogroup $B$ subunit vaccine and, if effective, will be an important inter- vention against this disease in epidemiological situations such as encountered in Brazil.

In conclusion, this is the first active hospital-based surveillance study of meningococcal meningitis in Brazil since the 1970s. It confirms that the epidemiological features of meningococcal meningitis have changed dramatically over the past three decades in Brazil, with the disappearance of large-scale epidemics caused by serogroups $A$ and $C$ and the introduction and establishment of serogroup B strains of the ET-5 complex (Sacchi et al., 2001). This study demonstrates that potentially important differences exist between the epidemiological features of meningococcal disease in Brazil and the developed world. Improved understanding of these differences and of the changing trends in the epidemiological features of this disease is essential to plan and implement strategies in order to prevent and reduce the high morbidity and mortality caused by $N$. meningitidis. It is noteworthy that the active hospital-

Table 3 Serotype:serosubtype distribution of Neisseria meningitidis isolates identified during active surveillance in Salvador, Brazil, between February 1996 and January 2001

\begin{tabular}{ll}
\hline Serotype:serosubtype & No. $(\%)$ of isolates $(n=377)$ \\
\hline $4,7: \mathrm{P} 1.19,15$ & $240(63.7)$ \\
$4,7: \mathrm{P} 1.7,1$ & $14(3.7)$ \\
$4,7: \mathrm{P} 1.3$ & $10(2.7)$ \\
$2 \mathrm{a}: \mathrm{P} 1.2$ & $9(2.4)$ \\
$2 \mathrm{a}: \mathrm{P} 1.5,2$ & $6(1.6)$ \\
2b:P1.5 & $5(1.3)$ \\
$4,10: \mathrm{P} 1.9$ & $5(1.3)$ \\
$4,7: \mathrm{nt}$ & $5(1.3)$ \\
$7: \mathrm{P} 1.19,15$ & $5(1.3)$ \\
Other & $64(17.0)$ \\
Non-typeable & $14(3.7)$ \\
\hline
\end{tabular}

a Other includes serotype:serosubtype strains isolated at a frequency of $<1 \%$. 
based surveillance became continuous and has provided valuable information regarding meningococcal meningitis today.

Authors' contributions: MGR and AIK conceived the idea for the project; SMC, ELG, JNR and AlK designed the study protocol; ABN, CTR, TSL, ELG and GSR performed the clinical evaluations and patient interviews; JNR, KMS and SMC performed the laboratory analyses; SMC, CTR, ABN, MLP and ELG performed the data analysis; SMC, ABN and MLP drafted the manuscript and JNR, MGR and AIK made the final revision. All authors read and approved the final manuscript. AlK is guarantor of the paper.

Acknowledgements: We thank the clinical, laboratory and administrative staff of Couto Maia Hospital, especially Ana Maria Maia and Neide Oliveira Silva; Steve Copolla and Ricardo Martinez Pinheiro for assistance in data collection and processing; Maviany Mota for assistance with laboratory analyses; Brendan Flannery and Luciano K. Silva for assistance with statistical analyses; Edgar Lessa Crusoé, Orgalí Marques, Neci Ivo Ramos and Nilda Lúcia Nunes Ivo from Bahia Secretary of Health for providing information on meningitis case notifications; Ana Paula S. Lemos from São Paulo Secretary of Health for providing information about serotype and serosubtype; and Lee W. Riley and Warren D. Johnson Jr for critical advice during study implementation. Most of all we thank the study patients and their families.

Funding: Grants from the Brazilian National Research Council $(300.861 / 96)$ and National Institutes of Health, USA (TW-00919, TW-007303).

Conflicts of interest: None declared.

Ethical approval: The Institutional Review Boards of the Oswaldo Cruz Foundation, Brazilian Ministry of Health in Salvador, Bahia, Brazil, and Weill Medical College of Cornell University in New York, NY, USA.

\section{References}

Berry, G., Armitage, P., 1995. Mid-P confidence intervals: a brief review. Statistician 44, 417-423.

Bilukha, O.O., Rosenstein, N., 2005. Prevention and control of meningococcal disease. Recommendations of the Advisory Committee on Immunization Practices (ACIP). MMWR Recomm. Rep. $54,1-21$

Bjune, G., Hoiby, E.A., Gronnesby, J.K., Arnesen, O., Fredriksen, J.H., Lindbak, A.K., Nokleby, H., Rosenqvist, E., Solberg, L.K. Closs, O., Eng, J., Froholm, L.O., Lystad, A., Bakketeig, L.S., Hareide, B., Halstensen, A., Holten, E., Eng, J., 1991. Effect of outer membrane vesicle vaccine against group $B$ meningococcal disease in Norway. Lancet 338, 1093-1096.

Brazilian Institute for Geography and Statistics, 2002. Anuário estatístico do Brasil, 2000. Rio de Janeiro, IBGE.

Cartwright, K., Noah, N., Peltola, H., 2001. Meningococcal disease in Europe: epidemiology, mortality, and prevention with conjugate vaccines. Report of a European advisory board meeting Vienna, Austria, 6-8 October, 2000. Vaccine 19, 4347-4356.

Caugant, D.A., 1998. Population genetics and molecular epidemiology of Neisseria meningitidis. APMIS 106, 505-525.
Caugant, D.A., Froholm, L.O., Bovre, K., Holten, E., Frasch, C.E., Mocca, L.F., Zollinger, W.D., Selander, R.K., 1987. Intercontinental spread of Neisseria meningitidis clones of the ET-5 complex. Antonie Van Leeuwenhoek 53, 389-394.

Costa, E.A., Martins, H., Klein, C.H., 1996. Evaluation of the protection received by a $B C$ antimeningococcal vaccine in the State of Santa Catarina, Brazil, 1990/92. Rev. Saude Publica 30, 460-470.

De Moraes, J.C., Camargo, M.C., Hidalgo, N.T., Barbosa, H.A., Gattas, V.L., Vasconcelos, H.G., Sacchi, C.T., Land Gral, I.M., Perkins, B.A., Wenger, J.D., Plikaytis, B.D., Broome, C.V., 1992. Protective efficacy of a serogroup $B$ meningococcal vaccine in Sao Paulo, Brazil. Lancet 340, 1074-1078.

Diermayer, M., Hedberg, K., Hoesly, F., Fischer, M., Perkins, B., Reeves, M., Fleming, D., 1999. Epidemic serogroup B meningococcal disease in Oregon: the evolving epidemiology of the ET-5 strain. JAMA 281, 1493-1497.

Edwards, M.S., Baker, C.J., 1981. Complications and sequelae of meningococcal infections in children. J. Pediatr. 99, 540-545.

Flexner, S., 1913. The results of serum treatment in thirteen hundred cases of epidemic meningitis. J. Exp. Med. 106, 553-576.

Giuliani, M.M., Adu-Bobie, J., Comanducci, M., Arico, B., Savino, S., Santini, L., Brunelli, B., Bambini, S., Biolchi, A., Capecchi, B., Cartocci, E., Ciucchi, L., Di Marcello, F., Ferlicca, F., Galli, B., Luzzi, E., Masignani, V., Serruto, D., Veggi, D., Contorni, M., Morandi, M., Bartalesi, A., Cinotti, V., Mannucci, D., Titta, F., Ovidi, E., Welsch, J.A., Granoff, D., Rappuoli, R., Pizza, M., 2006. A universal vaccine for serogroup $B$ meningococcus. Proc. Natl. Acad. Sci. USA 103, 10834-10839.

Jackson, L.A., Wenger, J.D., 1993. Laboratory-based surveillance for meningococcal disease in selected areas, United States, 1989-1991. MMWR CDC Surveill. Summ. 42, 21-30.

Jensen, E.S., Lundbye-Christensen, S., Pedersen, L., Sørensen, H.T., Schønheyder, H.C., 2003. Seasonal variation in meningococcal disease in Denmark: relation to age and meningococcal phenotype. Scand. J. Infect. Dis. 35, 226-229.

Kirsch, E.A., Barton, R.P., Kitchen, L., Giroir, B.P., 1996. Pathophysiology, treatment and outcome of meningococcemia: a review and recent experience. Pediatr. Infect. Dis. J. 15, 967-978, quiz 979.

Lemos, A.P., Brandao, A.P., Gorla, M.C., Paiva, M.V., Simonsen, V., Melles, C.E., 2006. Phenotypic characterization of Neisseria meningitidis strains isolated from invasive disease in Brazil from 1990 to 2001. J. Med. Microbiol. 55, 751-757.

Lind, I., Berthelsen, L., 2005. Epidemiology of meningococcal disease in Denmark 1974-1999: contribution of the laboratory surveillance system. Epidemiol. Infect. 133, 205-215.

Masignani, V., Comanducci, M., Giuliani, M.M., Bambini, S., AduBobie, J., Arico, B., Brunelli, B., Pieri, A., Santini, L., Savino, S., Serruto, D., Litt, D., Kroll, S., Welsch, J.A., Granoff, D.M., Rappuoli, R., Pizza, M., 2003. Vaccination against Neisseria meningitidis using three variants of the lipoprotein GNA1870. J. Exp. Med. 197, 789-799.

Peltola, H., 1983. Meningococcal disease: still with us. Rev. Infect. Dis. 5, 71-91.

Pollard, A.J., Levin, M., 2000. Vaccines for prevention of meningococcal disease. Pediatr. Infect. Dis. J. 19, 333-344, quiz 345.

Poolman, J.T., Lind, I., Jonsdottir, K., Froholm, L.O., Jones, D.M., Zanen, H.C., 1986. Meningococcal serotypes and serogroup B disease in north-west Europe. Lancet 2, 555-558.

Rosenstein, N.E., Perkins, B.A., Stephens, D.S., Lefkowitz, L., Cartter, M.L., Danila, R., Cieslak, P., Shutt, K.A., Popovic, T., Schuchat, A., Harrison, L.H., Reingold, A.L., 1999. The changing epidemiology of meningococcal disease in the United States, 1992-1996. J. Infect. Dis. 180, 1894-1901.

Rosenstein, N.E., Perkins, B.A., Stephens, D.S., Popovic, T., Hughes, J.M., 2001. Meningococcal disease. N. Engl. J. Med. 344, $1378-1388$. 
Meningococcal meningitis in Brazil

1153

Sacchi, C.T., Zanella, R.C., Caugant, D.A., Frasch, C.E., Hidalgo, N.T., Milagres, L.G., Pessoa, L.L., Ramos, S.R., Camargo, M.C., Melles, C.E., 1992. Emergence of a new clone of serogroup C Neisseria meningitidis in Sao Paulo, Brazil. J. Clin. Microbiol. 30, 1282-1286.

Sacchi, C.T., Lemos, A.P., Popovic, T., De Morais, J.C., Whitney, A.M., Melles, C.E., Brondi, L.M., Monteiro, L.M., Paiva, M.V., Solari, C.A., Mayer, L.W., 2001. Serosubtypes and PorA types of Neisseria meningitidis serogroup $B$ isolated in Brazil during 1997-1998: overview and implications for vaccine development. J. Clin. Microbiol. 39, 2897-2903.

Sierra, G.V., Campa, H.C., Varcacel, N.M., Garcia, I.L., Izquierdo, P.L., Sotolongo, P.F., Casanueva, G.V., Rico, C.O., Rodriguez, C.R., Terry, M.H., 1991. Vaccine against group B Neisseria meningitidis: protection trial and mass vaccination results in Cuba. NIPH Ann. 14, 195-207, discussion 208-210.

SVS/MS, 2005. Série histórica de casos de doenças de notificação compulsória no Brasil (1980-2005). Secretaria de Vigilância em Saúde, Ministério da Saúde, Brazil.

Tappero, J.W., Lagos, R., Ballesteros, A.M., Plikaytis, B., Williams, D., Dykes, J., Gheesling, L.L., Carlone, G.M., Hoiby, E.A., Holst, J., Nokleby, H., Rosenqvist, E., Sierra, G., Campa, C., Sotolongo,
F., Vega, J., Garcia, J., Herrera, P., Poolman, J.T., Perkins, B.A., 1999. Immunogenicity of 2 serogroup $B$ outer-membrane protein meningococcal vaccines: a randomized controlled trial in Chile. JAMA 281, 1520-1527.

Trotter, C.L., Andrews, N.J., Kaczmarski, E.B., Miller, E., Ramsay, M.E., 2004. Effectiveness of meningococcal serogroup C conjugate vaccine 4 years after introduction. Lancet 364, 365-367.

Tsolia, M.N., Theodoridou, M., Tzanakaki, G., Vlachou, V., Mostrou, G., Stripeli, F., Kalabalikis, P., Pangalis, A., Kafetzis, D., Kremastinou, J., Konstantopoulos, A., 2006. Invasive meningococcal disease in children in Greece: comparison of serogroup A disease with disease caused by other serogroups. Eur. J. Clin. Microbiol. Infect. Dis. 25, 449-456.

Wedege, E., Hoiby, E.A., Rosenqvist, E., Froholm, L.O., 1990. Serotyping and subtyping of Neisseria meningitidis isolates by co-agglutination, dot-blotting and ELISA. J. Med. Microbiol. 31, 195-201.

Wedege, E., Hoiby, E.A., Rosenqvist, E., Bjune, G., 1998. Immune responses against major outer membrane antigens of Neisseria meningitidis in vaccinees and controls who contracted meningococcal disease during the Norwegian serogroup B protection trial. Infect. Immun. 66, 3223-3231. 